\title{
Estimating insulin sensitivity and beta cell function: perspectives from the modern pandemics of obesity and type 2 diabetes
}

\author{
J. J. Nolan • K. Færch
}

Received: 8 February 2012 / Accepted: 18 May 2012 /Published online: 22 August 2012

(C) Springer-Verlag 2012

\begin{abstract}
The 1985 manuscript describing the HOMA model, by Matthews and colleagues, is the most cited paper in the history of Diabetologia. In this edition of 'Then and now' we assess the impact of this seminal paper by considering the contribution of this elegant work in the context of the most rapidly changing period in the history of diabetes. HOMA was born in the middle of an 'era' of insulin resistance, and was subsequently nurtured and grew during the 'eras' of insulin sensitisers and diabetes prevention. From the modern era of insulin resistance onward, researchers have sought a convenient method for measuring insulin sensitivity and secretion, and found this in HOMA. However, the explosion in the prevalence of diabetes clearly underlines that an understanding of insulin resistance and how it can be measured has been insufficient to make any impact on the growing pandemic of diabetes. Knowledge of individual physiology is important, but the dramatic impact of the modern environment may be the factor that has escaped attention until very recently. An optimist can only state that the coming 'era' in diabetes research will be a period of true translation of scientific insight and implementation of effective disease prevention.
\end{abstract}

J. J. Nolan $(\bowtie) \cdot$ K. Færch

Steno Diabetes Center A/S,

Niels Steensens Vej 2,

DK-2820 Gentofte, Denmark

e-mail: jjnl@steno.dk
Keywords Diabetes · Insulin resistance · Insulin secretion · Mathematical model $\cdot$ Obesity
Abbreviations
DPP
Diabetes Prevention Programme
GIP Glucose-dependent insulinotropic polypeptide
GLP-1 Glucagon-like peptide 1
HOMA-\%B
HOMA of beta cell function
HOMA-IR
HOMA of insulin resistance
IGT
Impaired glucose tolerance

\section{Challenges of a rapidly changing environment}

Over the last 50 years there has been an explosion in the number of diabetes cases [1-3] (Fig. 1a). In the 1930s, the concept of insulin resistance in type 2 diabetes was first mooted. Since then, it has increasingly been recognised that, in addition to beta cell failure, insulin resistance contributes significantly to the pathogenesis of type 2 diabetes. However, how these physiological principles of insulin resistance and gradual decline of endogenous insulin secretion could be measured and interpreted in humans remained a major challenge.

In 1985, 20 years after the publication of the first issue of Diabetologia, David R. Matthews and colleagues published a seminal research paper on a new structural model-based approach (HOMA) for the measurement of insulin resistance and beta cell function in humans [4]. This paper has been the most cited paper in the history of Diabetologia, with more than 8,600 citations to date (Fig. 1b). It is a privilege to reflect on the impact of this landmark publication 

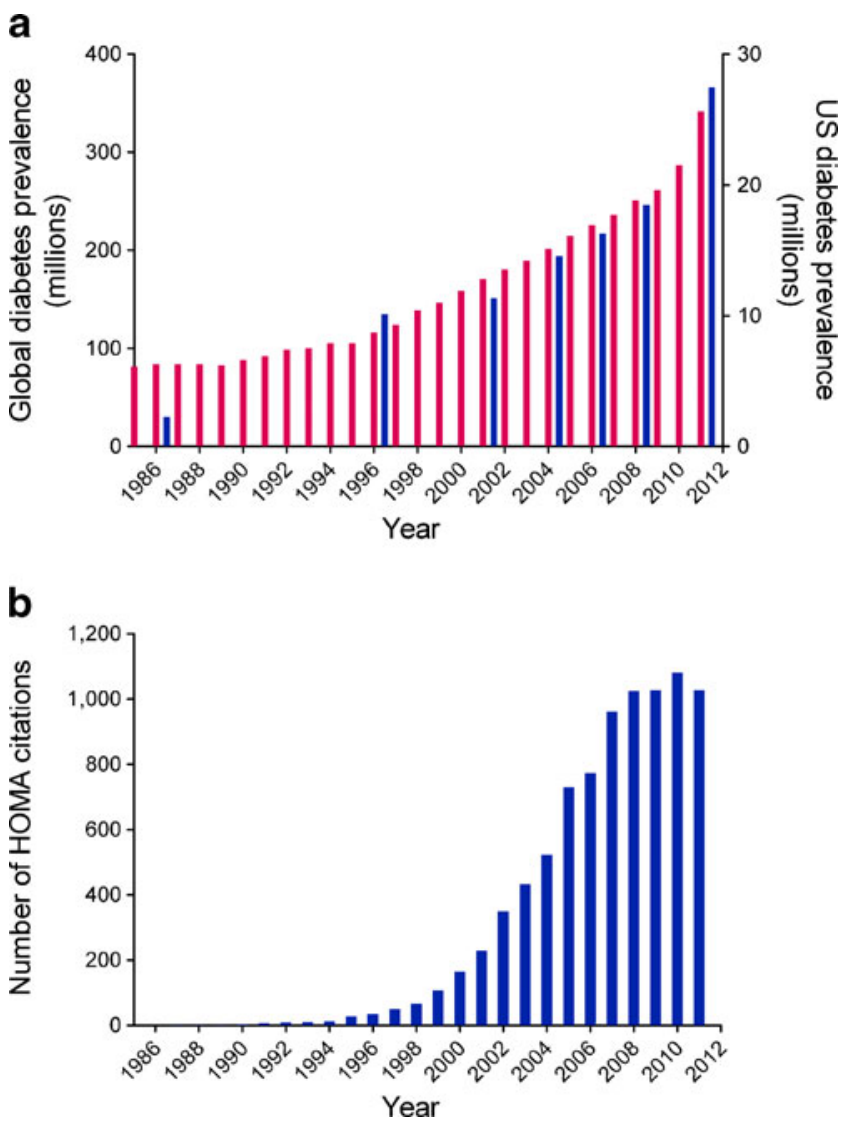

Fig. 1 (a) The trends in the global (blue) and US (red) prevalence of diabetes. (b) The number of citations of the paper by Matthews et al [4] from 1985 to 2011. Source: World Health Organization, International Diabetes Federation, Centers for Disease Control and Prevention, and Diabetologia Editorial Office

('Then'), and to place it in the context of the challenges of present day diabetes research and clinical care ('Now'). The paper by Matthews et al and the topic of HOMA in general have been extensively reviewed and discussed by experts in the field over the years [5-9], including Matthews and his colleagues [10], and it is not our purpose here to provide yet another critique or systematic review of HOMA. Instead we will consider the impact of this classic paper in the context of the overlapping modern 'eras' in diabetes research and care that have provided the backdrop to this scientific study (Fig. 2).

\section{Modern 'eras' in diabetes clinical research and care}

The 1985 HOMA paper [4] was published during the first of a series of 'eras' that have defined modern diabetes clinical research and care: the era of insulin resistance. This era was led by the insights and work of Gerald Reaven. Reaven was the first to clearly define the place of insulin resistance in the pathogenesis of type 2 diabetes, and later went on to describe the relevance of insulin resistance to the broader metabolic syndrome [11]. During this period the hyperinsulinaemic glucose clamp technique was described [12] and subsequently became the 'gold standard' for measurement of insulin sensitivity (Fig. 2). Moreover, the hyperglycaemic clamp was developed as a similar tool for estimation of insulin secretion. While the clamp technique became established as a reference method for estimation of insulin sensitivity and insulin secretion, it remained a restricted option and suitable only for those researchers with access to clinical investigation units, isotope tracers and substantial funding. In contrast, HOMA represented a relatively simple method for estimating insulin resistance and beta cell function from fasting plasma measurements of glucose and insulin.

The impact of the paper by Matthews et al [4] is clear from the trend in its annual number of citations. This increased from 1985, the date of its original publication, up to 2007, after which citations have plateaued at about 1,000 per year (Fig. 1b). From the beginning of the era of insulin resistance onward, it is clear that researchers have sought a convenient method for measuring insulin resistance, and appear to have found this in the HOMA of insulin resistance (HOMA-IR). On the other hand, HOMA has not been used to the same extent for the assessment of beta cell function (HOMA-\%B), despite there being a much higher level of general interest in beta cell function than insulin resistance among diabetes researchers (total number of PubMed publications in 2011: 11,955 vs 6,851, respectively). However, from the number of publications, it is obvious that general interest in insulin resistance has increased dramatically during the last decade (by $300 \%$ from 2000 to 2010), whereas the number of publications on beta cell function, has only increased by $\sim 50 \%$ during the same period. Thus, HOMAIR has filled a gap between a general increased interest and the need for a simple and convenient measure of insulin resistance in the diabetes community and in particular for those researching in diabetes epidemiology.

The second era of modern diabetes clinical research and care, which amplified the impact of the HOMA paper [4], was the era of insulin-sensitising treatments. This era began with the reintroduction of metformin to the US market in the early 1990s, and its use in the Diabetes Prevention Program (DPP) [13], which was funded by the US National Institutes of Health. The era was quickly established by the clinical characterisation of the PPAR- $\gamma$ agonists (glitazones), which have themselves gone through a rise and fall in use. More recently, bariatric surgery, both in terms of weight reduction and its effects on the underlying pathophysiology of type 2 diabetes, has overtaken all previous insulin-sensitising treatments in scale and potential impact. Thus, this era showed that insulin resistance can be treated both medically and surgically. 


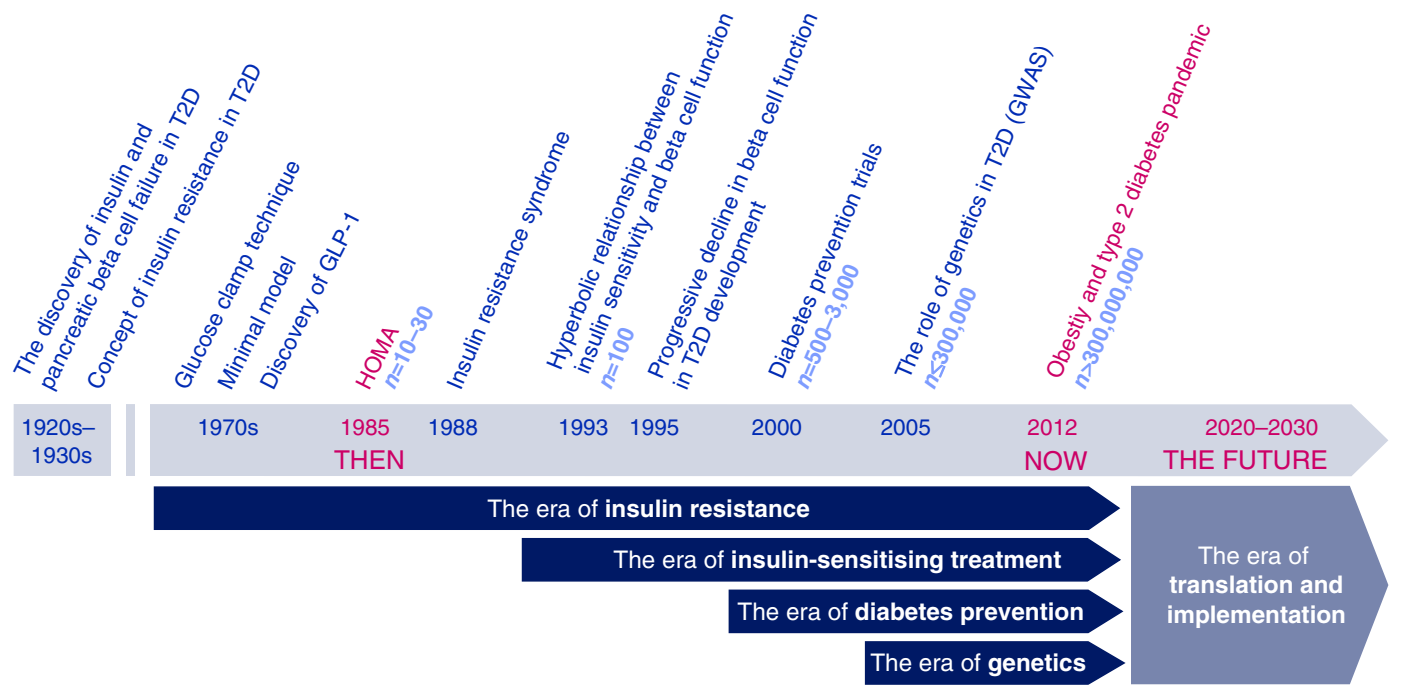

Fig. 2 A century of historic events that led to the development of the HOMA model and since then have added to our current understanding of the pathophysiology of type 2 diabetes (T2D). GWAS, genome-wide association studies

The third era was the era of diabetes prevention. The Finnish Diabetes Prevention Study, the DPP and several similar studies [13-16] proved that intensive diet and exercise intervention (as well as certain drugs) could prevent the onset of type 2 diabetes in individuals with impaired glucose tolerance (IGT). More recently, the results from a 3 year Japanese intervention study showed that individuals with isolated impaired fasting glycaemia (i.e. $2 \mathrm{~h}$ glucose $<7.8 \mathrm{mmol} / \mathrm{l}$ ) did not benefit in terms of diabetes risk from lifestyle modification [17], suggesting that pre-diabetes (impaired fasting glucose and/or impaired glucose tolerance) itself and diabetes prevention is more complex than was previously thought. However, the success of the landmark trials has not only consolidated the era of diabetes prevention, it has also advanced the field of insulin resistance and beta cell reserve protection in terms of measurement and treatment. This knowledge has yet to be translated or implemented to any effective extent on the world stage. Thus, a better understanding of insulin resistance and beta cell function, the measurement of both and how to treat them has not yet proved sufficient to make any impact on the growing pandemic of diabetes (Fig. 1a).

The most recent era, the era of genetics of chronic disease, has begun to explain the contribution of the human genome to obesity and type 2 diabetes. Most striking in this era is the large number of study participants required to prove significant relationships between common genetic variants and phenotypic traits (Fig. 2). To date, only a fraction of the diabetes phenotype can be accounted for by the diabetes genes that have been discovered. The majority of type 2 diabetes genes that have been identified relate to insulin secretion rather than insulin resistance [18]. Moreover, there appears to be discordance between the heritability of insulin resistance as measured by the clamp technique and by HOMA, consistent with differences in the basis for these two measurements of insulin resistance [19].

\section{Addressing the gap between clinical physiology and clinical practice}

The paper by Matthews et al [4] was published when there was a huge unmet need for a model to quantify both insulin resistance and beta cell function in diabetic and non-diabetic individuals. HOMA was originally devised as a simple mathematical algorithm for quantification of the relative contributions of insulin resistance and beta cell dysfunction to an individual's fasting hyperglycaemia. Given certain limitations [5-9], and despite the relatively small number of individuals studied to derive the 1985 model, HOMA has been widely accepted as a convenient surrogate marker of insulin resistance and beta cell function. To summarise expert critique, HOMA-IR has been well validated against the clamp technique and reflects hepatic more than peripheral insulin resistance, whereas HOMA-\%B has been less rigorously evaluated, and is a much less robust estimate of beta cell function $[5-9,20]$.

The increased interest in type 2 diabetes and the need for estimation of insulin resistance and beta cell function has led to an exponential increase in the use of HOMA (Fig. 1b). Many studies have focused on the statistical relationships between surrogate markers (especially HOMA) and clampderived markers of insulin sensitivity. However, lack of agreement between HOMA-IR and clamp-derived estimates of insulin resistance should not simply be interpreted as a weakness of HOMA-IR, but rather as a result of the different 
pathophysiological processes that are addressed by these different analytical tools (in particular, hepatic vs peripheral insulin resistance) [20]. More important now is the capacity of the different markers of insulin resistance or beta cell function to predict type 2 diabetes and cardiovascular disease in diverse populations. For these purposes HOMA may be useful; however, an index based only on fasting concentrations of glucose and insulin is subject to important limitations. One of the most notable differences between fasting and non-fasting measurements of insulin is that the incretin hormones glucagon-like peptide 1 (GLP-1) and glucosedependent insulinotropic polypeptide (GIP) are secreted in response to food intake and, hence, contribute significantly to glucose-dependent insulin secretion. In contrast, the effects of the incretin hormones are very limited during fasting conditions; thus, HOMA cannot account for the disturbances of incretin secretion and action that have been shown to be characteristic of both type 2 diabetes and prediabetes [21, 22].

Despite limitations that have been the subject of previous reviews [5-9], HOMA has endured owing to its solid foundations in physiology. In the original study [4], fasting insulin concentrations were measured from three samples over $10 \mathrm{~min}$, thereby taking account of the pulsatility of insulin secretion. The coefficient of variation between HOMA-\%B based on repeated samples was much smaller (CV 35\%) than that for a measure based on a single fasting sample (CV 52\%). Pulsatility of insulin is still not incorporated into most clinical investigations, and is certainly not routine clinical practice. A major positive development, however, is that the precision of insulin assays has much improved since the period when HOMA was developed (Now: CV 4-10\%, e.g. with AutoDELFIA; Then: CV 11$16 \%$ [23]), making single measurements of insulin much more reliable. There has been progress towards standardisation of the $\mathrm{HbA}_{1 \mathrm{c}}$ assay worldwide. Future studies diagnosing diabetes (or pre-diabetes) by $\mathrm{HbA}_{1 \mathrm{c}}$ may not measure fasting glucose and insulin concentrations. Thus, the new $\mathrm{HbA}_{1 \mathrm{c}}$-based approach to the diagnosis of diabetes and prediabetes may lead to a decline in the use of HOMA.

\section{HOMA in the current day context}

Today, when choosing a method for determination of insulin resistance and beta cell function, underlying physiological differences between measures should be taken into consideration. This choice is ultimately determined by the research question and the type of study. For large epidemiological studies the clamp technique is unsuitable because of its complexity and the associated time and costs. Either an oral glucose tolerance test or a meal tolerance test should be considered, since either provides important information about the underlying physiology beyond the fasting state. For more detailed physiology, HOMA on its own may be insufficient for estimation of insulin resistance and beta cell function but, when combined with nonfasting measures, may continue to provide useful and convenient information about glucose metabolism in the fasted state.

In 1998, 13 years after publication of the original HOMA paper [4], HOMA was updated with a computer model (HOMA2) based on non-linear solutions [10]. HOMA2 was proposed to provide a more accurate estimate of the physiological response to an intravenous glucose infusion and therefore to be preferable to the simple HOMA [24]. HOMA2 has been widely used and has replaced the original HOMA model for many researchers. However, it has not been convincingly demonstrated that HOMA2 is superior. One limitation is that the equation underlying this computer model has never been released. This is unfortunate and places HOMA2 somewhat out of step with the current focus on Open Access and data sharing policies. Publication of the underlying equation would make it possible for other researchers to validate the model and discuss whether (and how) the HOMA2 calculator is superior to the simple HOMA model as well as to other surrogate markers. Ultimately, development of simple computer models taking into account not only fasting glucose and insulin concentrations, but also other factors related to individual risk, may prove useful in future clinical practice.

\section{The future challenge of diabetes prevention and treatment}

Despite a better understanding of insulin resistance and beta cell dysfunction, as well as the development of sophisticated methods to measure and potentially treat insulin resistance in humans, there has been essentially no progress in the prevention of type 2 diabetes on a global scale during the period from 1965 to the present. In fact, the reverse is the case, as clearly illustrated in Fig. 1a. There has been an explosion in the prevalence of obesity and diabetes that has outpaced efforts to comprehend the disease and its scale. As illustrated in Fig. 2, the original HOMA algorithm was derived from a small number of study participants (about 30 in all). The subsequent diabetes prevention studies included from a few hundred to more than 3,000 individuals. The numbers needed to be studied to discover diabetes genes have been in the hundreds of thousands of individuals, and numbers currently affected by diabetes or pre-diabetes include hundreds of millions. Unfortunately, knowledge of individual insulin resistance and absolute or relative risk appears to have little impact on prevention. Preventing future diabetes cases on the broader scale clearly calls for 
insight into the underlying differences between subgroups at risk, in combination with national or international action plans.

Indeed, the challenge now is to implement research findings from recent decades-by translating physiology and biological insight into clinical practice - and into the daily life of millions of people in modern societies, particularly in the developing world. The optimistic view is that the next era of diabetes research will be one of successful translation and implementation.

Acknowledgments We thank Giovanni Pacini of the Metabolic Unit, ISIB-CNR, Padova, Italy for his advice and careful reading of this manuscript.

Contribution statement JJN and KF were responsible for interpretation of data and literature, drafting of the manuscript and approval of the final version for publication.

Duality of interest JJN and KF are employed by Steno Diabetes Center A/S, a diabetes research hospital working in the Danish National Health Service and wholly owned by Novo Nordisk A/S.

\section{References}

1. National Institute of Diabetes and Digestive and Kidney Disorders. www.win.niddk.nih.gov/statistics/index.htm\#overweight. Accessed April 2012

2. Flegal KM, Carroll MD, Ogden CL, Curtin LR (2010) Prevalence and trends in obesity among US Adults, 1999-2008. JAMA 303:235-241

3. International Diabetes Federation (2011) IDF Diabetes Atlas, 5th edn. IDF, Brussels

4. Matthews DR, Hosker JP, Rudenski AS, Naylor BA, Treacher DF, Turner RC (1985) Homeostasis model assessment: insulin resistance and beta-cell function from fasting plasma glucose and insulin concentrations in man. Diabetologia 28:412-419

5. McAuley KA, Mann JI, Chase JG, Lotz TF, Shaw GM (2007) Point: HOMA - satisfactory for the time being. HOMA: the best bet for the simple determination of insulin sensitivity, until something better comes along. Diabetes Care 30:2411-2413

6. Hockaday D, Sayyad M, Yajnik C (2007) Counterpoint: appreciating homeostasis model assessment. More useful earlier rather than later. Diabetes Care 30:2414-2418

7. Boyko EJ, Jensen CC (2007) Do we know what homeostasis model assessment measures? If not, does it matter? Diabetes Care $30: 2725-2728$
8. Ferrannini E, Mari A (1998) How to measure insulin sensitivity. J Hypertens 16:895-906

9. Buchanan TA, Watanabe RM, Xiang AH (2010) Limitations in surrogate measures of insulin resistance. J Clin Endocrinol Metab 95:4874-4876

10. Wallace TM, Levy JC, Matthews DR (2004) Use and abuse of HOMA modeling. Diabetes Care 27:1487-1495

11. Reaven GM (1988) Banting lecture 1988. Role of insulin resistance in human disease. Diabetes 37:1595-1607

12. DeFronzo RA, Tobin JD, Andres R (1979) Glucose clamp tecnique: a method for quantifying insulin secretion and resistance. Am J Physiol 237:E214-E223

13. Knowler WC, Barrett-Connor E, Fowler SE et al (2002) Reduction in the incidence of type 2 diabetes with lifestyle intervention or metformin. N Engl J Med 346:393-403

14. Tuomilehto J, Lindström J, Eriksson JG et al (2001) Prevention of type 2 diabetes mellitus by changes in lifestyle among subjects with impaired glucose tolerance. N Engl J Med 344:1343-1350

15. Pan XR, Li GW, Hu YH et al (1997) Effects of diet and exercise in preventing NIDDM in people with impaired glucose tolerance. The Da Qing IGT and Diabetes Study. Diabetes Care 20:537-544

16. Eriksson KF, Lindgärde F (1991) Prevention of type 2 (non-insulindependent) diabetes mellitus by diet and physical exercise. The 6year Malmö feasibility study. Diabetologia 34:891-898

17. Saito T, Watanabe M, Nishida J et al (2011) Lifestyle modification and prevention of type 2 diabetes in overweight Japanese with impaired fasting glucose levels: a randomized controlled trial. Arch Intern Med 171:1352-1360

18. Florez J (2008) Newly identified loci highlight beta cell dysfunction as a key cause of type 2 diabetes: Where are the insulin resistance genes? Diabetologia 51:1100-1110

19. Rasmussen-Torvik LJ, Pankow JS, Jacobs DR et al (2007) Heritability and genetic correlations of insulin sensitivity measured by the euglycaemic clamp. Diabet Med 24:1286-1289

20. Abdul-Ghani MA, Matsuda M, Balas B, DeFronzo RA (2007) Muscle and liver insulin resistance indexes derived from the oral glucose tolerance test. Diabetes Care 30:89-94

21. Nauck MA, Vardarli I, Deacon CF, Holst JJ, Meier JJ (2011) Secretion of glucagon-like peptide-1 (GLP-1) in type 2 diabetes: what is up, what is down? Diabetologia 54:10-18

22. Færch K, Vaag A, Holst J, Glümer C, Pedersen O, Borch-Johnsen K (2008) Impaired fasting glycaemia vs impaired glucose tolerance: similar impairment of pancreatic alpha and beta cell function but differential roles of incretin hormones and insulin action. Diabetologia 51:853-861

23. Hosker JP, Matthews DR, Rudenski AS et al (1985) Continuous infusion of glucose with model assessment: measurement of insulin resistance and beta-cell function in man. Diabetologia 28:401-411

24. Levy JC, Matthews DR, Hermans MP (1998) Correct homeostasis model assessment (HOMA) evaluation uses the computer program. Diabetes Care 21:2191-2192 